\title{
A Current-Mode CMOS Integrated Microsystem for Current Spinning Magnetic Hall Sensors
}

\author{
Hadi Heidari, Edoardo Bonizzoni, Umberto Gatti, and Franco Maloberti \\ Department of Electrical, Computer, and Biomedical Engineering \\ Via Ferrata, 1 - 27100 Pavia - ITALY \\ E-mails: \{hadi.heidari, edoardo.bonizzoni, franco.maloberti\}@unipv.it, gattiu@alice.it
}

\begin{abstract}
A magnetic Hall sensor working in the currentmode is presented. The proposed sensing device is composed by two Hall plates able to provide a differential current at the output nodes. The sensor, fabricated in a standard 0.18- $\mu \mathrm{m}$ CMOS technology, uses the spinning-current technique to compensate for the offset and obtains a sensitivity $\mathbf{I}_{\text {Hall }} /\left(\mathbf{B}_{\perp} \mathbf{I}_{\text {bias }}\right)$ better than $0.02 \mathrm{~T}^{-1}$ for magnetic fields ranging from 0 to $10 \mathrm{mT}$.
\end{abstract}

\section{INTRODUCTION}

A magnetic Hall sensor is a transducer, which converts a magnetic field into a corresponding electrical signal. Magnetic Hall micro-sensors generally use integrated Hall plates as sensing devices, which are fully compatible with standard CMOS technologies. Hall effect sensors are widely used for proximity switching, positioning, speed detection, and current sensing applications.

Typically, Hall plates are used in voltage-mode and for many years voltage-mode Hall sensors have been absolutely dominant in most of the applications. This means that the magnetic field to be measured is converted into an output voltage. Signal-to-noise ratio (SNR) and offset are important features in Hall sensors performance evaluation. Several techniques have been developed in order to improve these characteristics. Most of them, [1]-[5], are based on conventional voltage-mode Hall transducers. Parasitic capacitances and their effect, specially at high frequency operation, affect performance of voltage-mode solutions.

As an alternative solution, an Hall sensor can work in the current mode. The output is current and not voltage. In the current-mode Hall sensors there is no variation of the terminal potentials and so no influence of the parasitic capacitances. Also, it can be possible to use of smaller number of terminals, therefore the ultimate miniaturization of the device is easier, [1].

This paper presents a low power current-mode Hall sensor microsystem with differential output current, as shown in Fig. 1. The physical structure of the proposed current-mode Hall sensor is the same of modern devices, with the same possibility of compensating for the offset caused by mismatch (current spinning). The difference is in driving and extracting the signal. Extensive and accurate physical simulations and behavioral models of the current-mode Hall sensors allowed optimizing its size, shape and performance for the chosen technology, a standard 0.18- $\mu \mathrm{m}$ CMOS technology. The offset

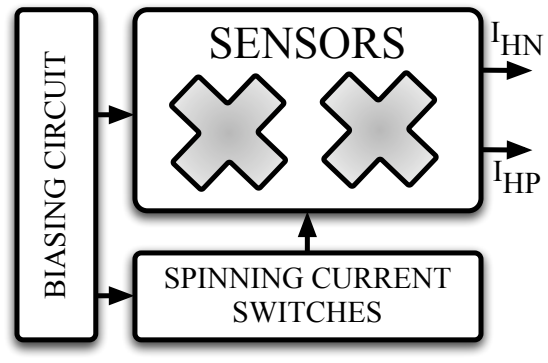

Fig. 1. The block diagram of the proposed magnetic current-mode Hall sensor.

at the output terminals of the sensors is eliminated by spinning current technique and measurement results show good sensitivity better than $0.02 \mathrm{~T}^{-1}$ for magnetic fields in the range from 0 to $10 \mathrm{mT}$. The circuit operates properly with a bias current as low as $12 \mu \mathrm{A}$. The benefit of having current at output is that its integration over a defined time period determines an output voltage with a gain proportional to the integration time.

The paper is organized as follows. In Section II the current-mode technique is presented, discussed and compared with the voltage-mode counterpart. Section III describes the microsystem implementation, while measurement results are presented in section IV. Section V draws some conclusions.

\section{Current-Mode Hall Sensors}

The description of an Hall sensor working in the voltagemode can be found in [1] and [3]. When operating in this mode, the Hall voltage $\left(\mathrm{V}_{\text {Hall }}\right)$ is proportional to the sensor bias current, $\mathrm{I}_{\text {bias }}$, the current-related voltage-mode sensitivity, $S_{V}$, and the applied perpendicular external magnetic field, $\mathrm{B}_{\perp}$,

$$
V_{\text {Hall }}=S_{V} I_{\text {bias }} B_{\perp}
$$

The current-mode Hall sensor principle has been described in [6]. In this microsystem, the method is applied to a couple of Hall plates in order to achieve differential currents at the output.

Fig. 2 shows the proposed current-mode Hall sensor. The basic idea consists in injecting a current, $\mathrm{I}_{B 1}=\mathrm{I}_{\text {bias }}$, laterally in two consecutive arms of the first cross-shaped Hall plate and draining nominally the same current, $\mathrm{I}_{B 2}$, from two consecutive 


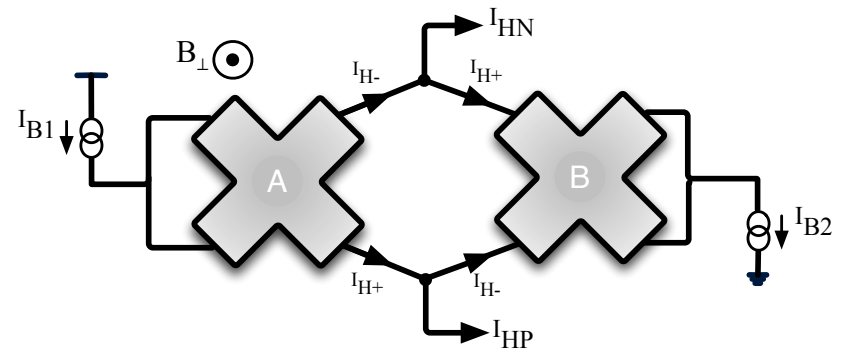

Fig. 2. Proposed current-mode Hall sensor structure.

arms of the second Hall plate. In this condition, with zero magnetic field the sensor structure is balanced thus making null the output currents, $\mathrm{I}_{H N}$ and $\mathrm{I}_{H P}$. An external magnetic field unbalances the sensor, providing two Hall currents, $I_{\text {Hall }}$, at the output nodes. The Hall current, for a cross-shaped Hall plate, can be expressed as [6]

$$
I_{\text {Hall }}=\frac{\beta B_{\perp} I_{\text {bias }}}{1-\left(\beta B_{\perp}\right)^{2}}
$$

where $\beta$ is the magnetic resistance coefficient in presence of a magnetic field. It is defined as

$$
\beta=\frac{R_{(B)}-R_{(B=0)}}{R_{(B=0)} B_{\perp}}
$$

where $R_{(B=0)}$ and $R_{(B)}$ define the Hall plate resistance in absence and presence of an external magnetic field, respectively.

The output currents of each Hall plate, $\mathrm{I}_{H_{+}}$and $\mathrm{I}_{H_{-}}$in Fig. 2, are

$$
\begin{aligned}
& I_{H+}=\frac{I_{\text {bias }}}{2}+\frac{I_{\text {Hall }}}{2} \\
& I_{H-}=\frac{I_{\text {bias }}}{2}-\frac{I_{\text {Hall }}}{2}
\end{aligned}
$$

In the proposed configuration, the use of a couple of Hall plates makes the sensor output currents, $\mathrm{I}_{H P}$ and $\mathrm{I}_{H N}$, being the difference of the output currents of each Hall plate. Using equations (4) and (5), the sensor output currents are given by

$$
\begin{gathered}
I_{H P}=I_{H+}-I_{H-}=I_{\text {Hall }} \\
I_{H N}=I_{H-}-I_{H+}=-I_{\text {Hall }}
\end{gathered}
$$

The current-mode magnetic sensitivity is used to evaluated the performance of current-mode Hall sensors. It is defined as

$$
S_{I}=\frac{I_{\text {Hall }}}{I_{\text {bias }} B_{\perp}}
$$

For the used configuration, the difference of the output currents is $2 \times \mathrm{I}_{\text {Hall }}$, thus doubling the sensor sensitivity with respect to the conventional implementation.

\section{Circuit Implementation}

The proposed microsystem, shown in Fig. 1, consists of two magnetic Hall plates used as the sensing device, a number of switches to implement the current spinning and a biasing circuit. This Section describes the implementation details of each block.

\section{A. Current-Mode Hall Plates}

Several current-mode Hall structures have been modeled and evaluated with respect to noise, offset and sensitivity using COMSOL Multiphysics ${ }^{\mathrm{TM}}$ and Verilog-A, [6]. The crossshaped model is the optimum plate structure to fit the lowest noise and residual offset and the best sensitivity. In addition, the symmetrical cross-shaped Hall plate grants high sensitivity and immunity to alignment tolerances resulting from the fabrication process.

A low doped $\mathrm{N}$-well makes the Hall plates. In order to reduce the flicker noise and surface carrier losses, a shallow highly doped $\mathrm{P}^{+}$conductive top layer covers the surface of the active area. The four contact regions in the $\mathrm{N}$-well diffusion are $\mathrm{N}^{+}$highly doped.

Fig. 3 shows the model geometry of the two Hall plates used in a COMSOL Multiphysics ${ }^{\mathrm{TM}}$ simulation: the maximum width and length of each plate is $8 \mu \mathrm{m}$. The figure shows the simulated surface electrical distribution and the current distribution when a perpendicular magnetic field of $10 \mathrm{mT}$ is applied. The simulation, carried out with physical parameters fitting the used CMOS technology, uses the nominal bias current of $12 \mu \mathrm{A}$ injected in terminals 1 and 2 and drained from terminals 3 and 4 .

\section{B. Current Spinning}

Since the output offset strongly limits the DC resolution of the sensors, the use of the crossed-shaped Hall plate enables the current spinning technique, [7] [8], to reduce offset and $1 / f$ noise.

The output and supply terminals of each Hall plate are periodically interchanged so that the input bias current polarity is rotated in each state whereas the offset appears at the output terminals. The plates are clocked with four phases. At the

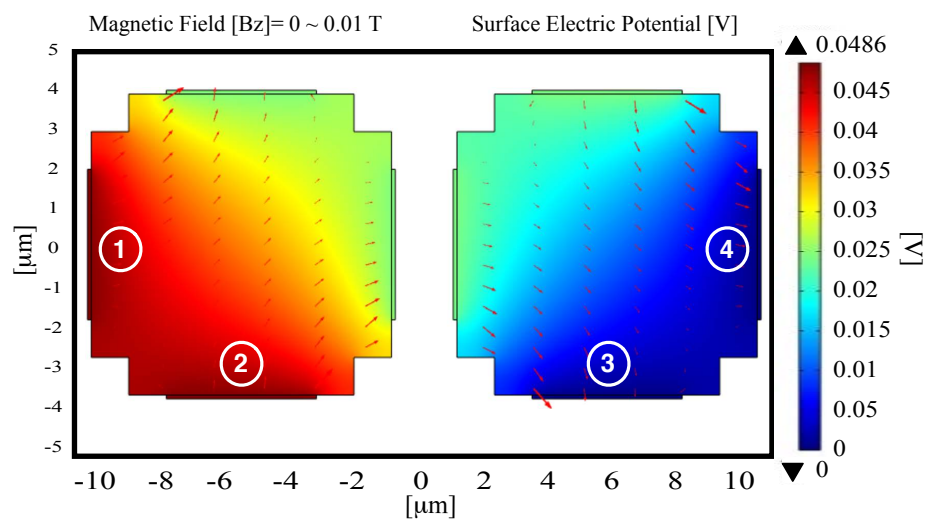

Fig. 3. COMSOL Multiphysics ${ }^{\mathrm{TM}}$ simulation results. 


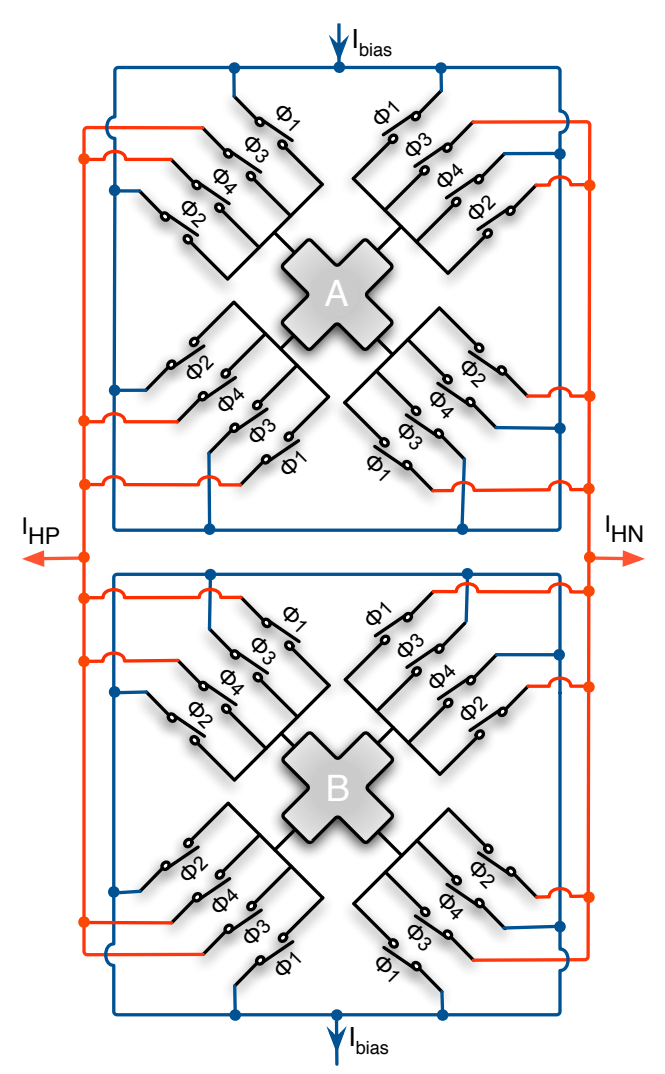

Fig. 4. Hall plates with switches configuration for current spinning.

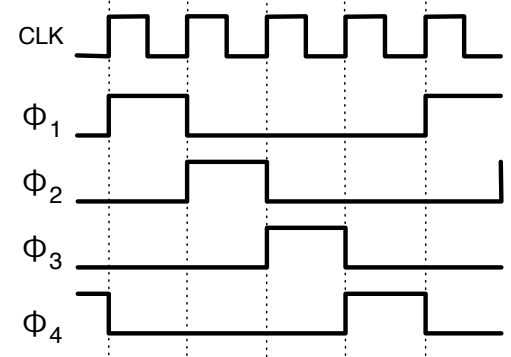

Fig. 5. Phases timing diagram for current spinning operation.

end of a complete spinning operation, the average of offset becomes zero.

Fig. 4 shows the sensor and switches configuration driven by the four phases whose time diagram is given in Fig. 5. During each phase, $\Phi_{1}, \Phi_{2}, \Phi_{3}$, and $\Phi_{4}$, for each plate two terminals are connected to $\mathrm{I}_{\text {bias }}$ while the remaining two are the outputs. The implementation uses 32 switches, each of them is a complementary CMOS pair.

\section{Biasing Circuit}

The sensor structure of Fig. 2 uses two current generators nominally equal. A possible mismatch brings the one with the larger value to the triode region. This imposes to use a feedback control to make equal the two current generators. Fig. 6 shows the used circuit. The P-channel cascade current mirror replicates the bias current, $\mathrm{I}_{\text {bias }}$. A second cascade

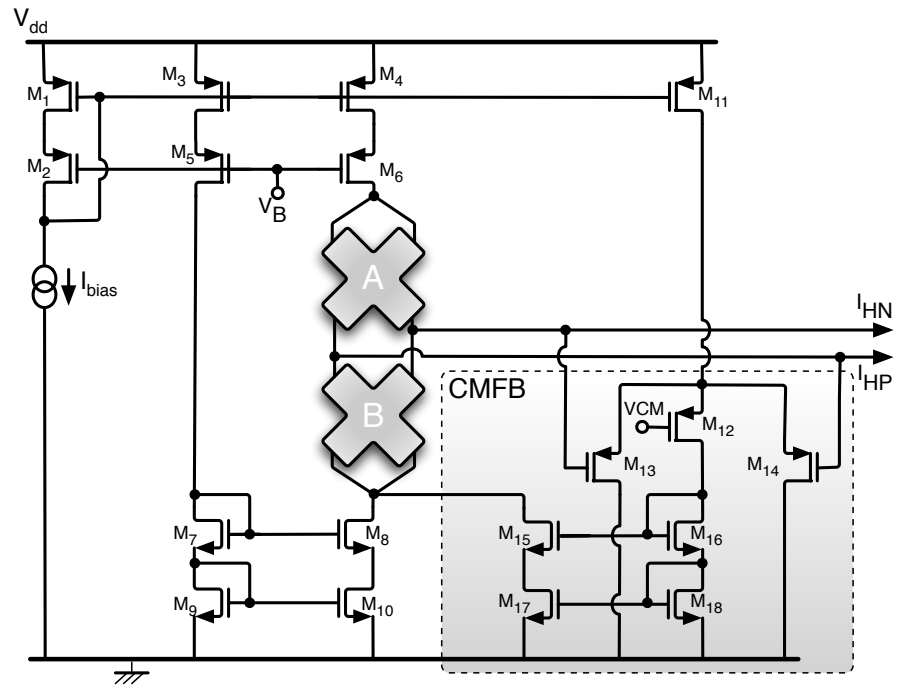

Fig. 6. Schematic diagram of Hall plates with biasing and common mode feedback circuit.

mirror gives rise to the sink current. Its value is systematically less than the required voltage to leave some room for the common mode control. This current is a P-channel differential pair sensitive to the common mode input. The current flowing in $\mathrm{M}_{12}$ mirrored by $\mathrm{M}_{15} \sim \mathrm{M}_{18}$ makes the common mode voltage at output of the sensor equal to VCM, thus ensuring that the drain and sink bias currents are equal.

\section{Measurement Results}

A sensor prototype has been fabricated in a standard $0.18-\mu \mathrm{m}$ CMOS process with 6 metal and 2 poly layers. Its microphotograph with its layout back-annotated is shown in Fig. 7. The figure highlights main circuital blocks. The microsystem occupies an active area of $80 \times 50 \mu \mathrm{m}^{2}$. The nominal supply voltage and the nominal bias current are $1.8 \mathrm{~V}$ and $12 \mu \mathrm{A}$, respectively. The current spinning frequency is $1 \mathrm{MHz}$.

A measurement setup is shown in Fig. 8. A digital signal generator/analyzer provides the four phases for spinning current. Helmholtz coils apply to the integrated microsystem a magnetic field ranging from 0 to $10 \mathrm{mT}$.

Fig. 9 plots the measured differential sensor output current, $\mathrm{I}_{\text {Hall }}$, as a function of the sensor bias current for two different

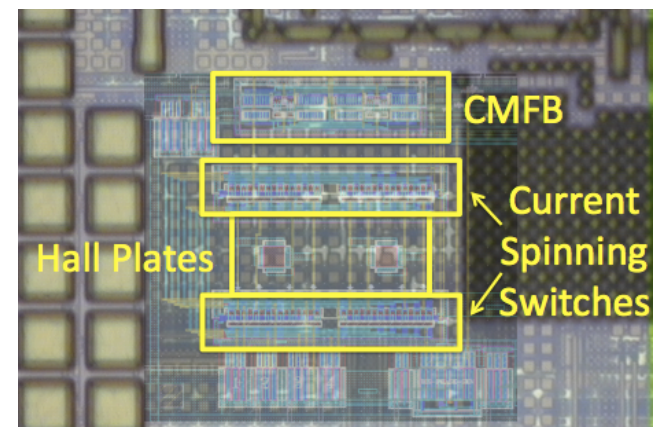

Fig. 7. Chip microphotograph with layout back annotation. 


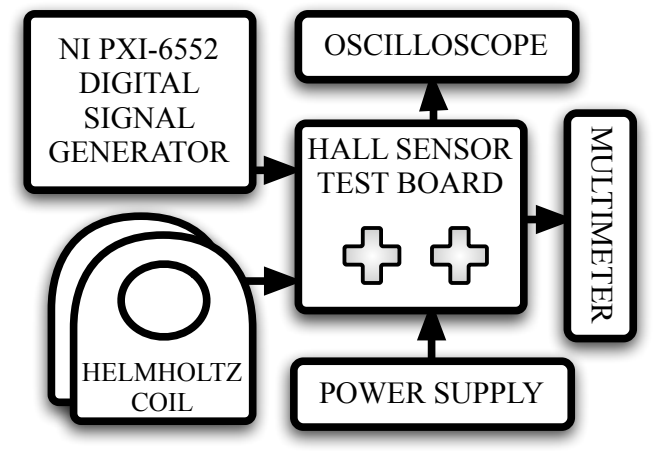

Fig. 8. Used measurement setup.

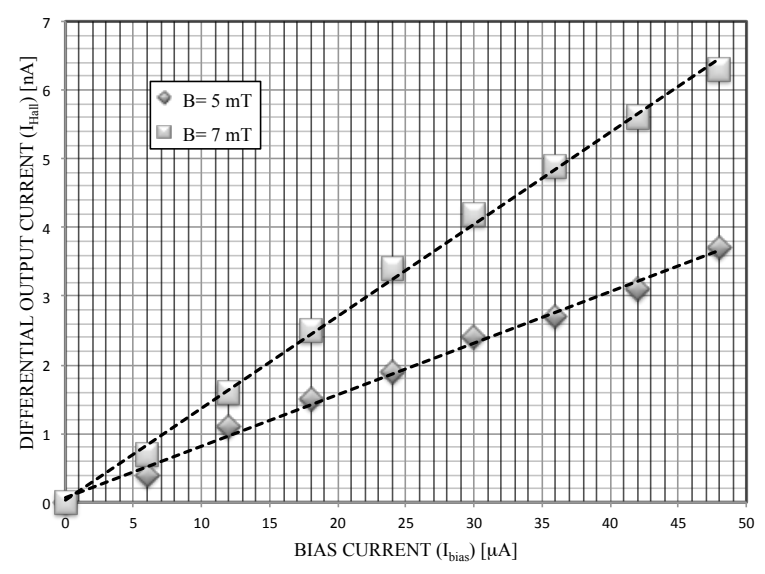

Fig. 9. Measured sensor output current as a function of the biasing current at different magnetic fields.

magnetic field, $5 \mathrm{mT}$ and $7 \mathrm{mT}$. $\mathrm{I}_{\text {bias }}$ is ranging from 0 to $48 \mu \mathrm{A}$. The figure shows that the sensor has a good linearity with respect to the applied magnetic field.

Fig. 10 gives the sensitivity, $S_{I}$, as a function of the sensor bias current (ranging from 6 to $48 \mu \mathrm{A}$ ) for the two above external magnetic fields. The measured sensor sensitivity is within the $0.013 \mathrm{~T}^{-1}-0.02 \mathrm{~T}^{-1}$ range.

Table I summarizes the magnetic and electrical microsystem performance.

TABle I. Performance Summary

\begin{tabular}{c||c}
\hline Technology & $0.18 \mu \mathrm{m} \mathrm{CMOS}$ \\
\hline Sensor Area & $80 \times 50 \mu \mathrm{m}^{2}$ \\
\hline Plate Area & $8 \times 8 \mu \mathrm{m}^{2}$ \\
\hline Number of Sensors & 2 \\
\hline Current Spinning Frequency & $1 \mathrm{MHz}$ \\
\hline Supply Voltage & $1.8 \mathrm{~V}$ \\
\hline Sensitivity & $0.013 \mathrm{~T}^{-1} \sim 0.02 \mathrm{~T}^{-1}$ \\
\hline Measurement Range & $0 \sim 10 \mathrm{mT}$ \\
\hline Power Consumption & $65 \mu \mathrm{W}$ \\
\hline
\end{tabular}

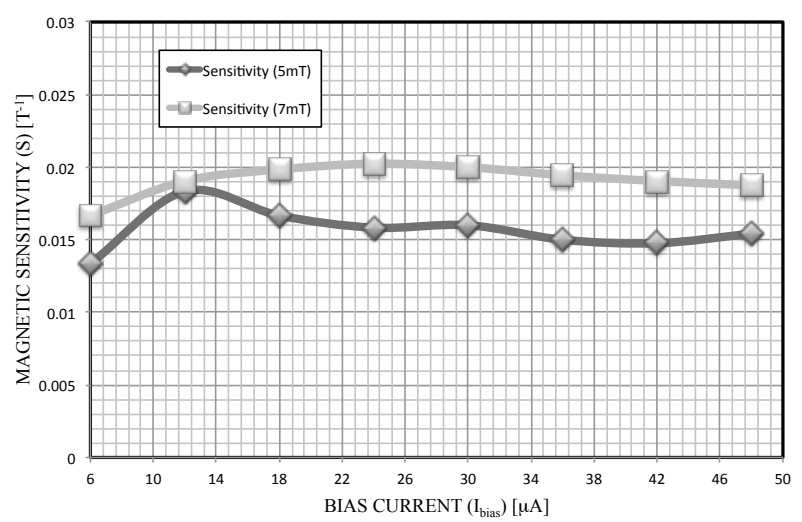

Fig. 10. Measured sensor sensitivity as a function of the biasing current at different magnetic fields.

\section{Conclusion}

This paper presented a magnetic current-mode Hall microsystem. Two Hall plates operating in the current-mode and able to provide differential currents at the output nodes has been fabricated in a standard $0.18-\mu \mathrm{m}$ CMOS process. Measurement results show that the Hall sensor achieves a sensitivity better than $0.02 \mathrm{~T}^{-1}$ when the magnetic field is in the range from 0 to $10 \mathrm{mT}$. The use of the crossed-shaped Hall plates and a current-mode approach enables current spinning technique for offset cancellation. The power consumption is in the tens of $\mu \mathrm{W}$ range. Moreover, this Hall device can be easily integrated with a readout circuit in a single chip to increase the magnetic sensitivity.

\section{REFERENCES}

[1] R. S. Popovic, Hall Effect Devices, 2nd ed. Bristol, U.K.: Inst. Physics Publishing, 2004.

[2] A. Ajbl, M. Pastre, and M. Kayal, "A Hall sensor microsystem with continuous gain calibration using fully integrated references", Proc. of IEEE International Symposium of Circuits and Systems (ISCAS), pp. 1399-1402, May 2012.

[3] M. Pastre, M. Kayal, and H. Blanchardl, "A Hall Sensor Analog Front End for Current Measurement with Continuous Gain Calibration", IEEE International Solid-State Circuits Conference (ISSCC) Dig. Tech. Pap., pp. 242-243, 596, Feb. 2005.

[4] G. Boero, D. Memierre, P.-.A. Besse and R.S. Popovic, "Micro-Hall devices: performance, technologies and applications", Sensors and Actuators $A$, no. 106, pp. 314-320, 2003

[5] Y.J. Min, Ch.K. Kwon, H.K. Kim, Ch. Kim and S.W. Kim, "A CMOS Magnetic Hall Sensor Using a Switched Biasing Amplifier", IEEE Sensors Journal, vol. 12, no. 5, pp. 1195-1196, May 2012.

[6] H. Heidari, U. Gatti, E. Bonizzoni and F. Maloberti, "Low-Noise LowOffset Current-Mode Hall Sensors", Proc. of Conference on Ph.D. Research in Microelectronics and Electronics (PRIME), pp. 325-328, June 2013.

[7] A. Bilotti, G. Monreal, and R. Vig, "Monolithic Magnetic Hall Sensor Using Dynamic Quadrature Offset Cancellation", IEEE Journal of SolidState Circuits, vol. 32, no. 6, pp. 829-836, June 1997.

[8] S. Bellekom and R. Bellekom and S. Yin and A. Bakker, "Spinningcurrent Hall plate with integrated switches", Proceedings of the ProRISC Workshop on Circuits, Systems and Signal Processing, pp. 37-41, 1997. 